\title{
Spontaneous abortion with MRI correlates in the emergency department
}

Spontaneous abortions are associated with 20 percent of all pregnancies and they are the most common cause of first trimester bleeding. Diagnosis is based on ultrasound findings. Patients typically present with vaginal bleeding and severe lower abdominal pain. We report a 25 year old female with vaginal bleeding, bilateral abdominal pain, and normal transvaginal US findings on admission. Follow-up magnetic resonance imaging in the emergency department demonstrated heterogeneous, T2 isointense/hyperintense signal material extending from the uterine cavity and protruding into the vaginal canal, consistent with active passage of products of conception. Findings were confirmed by pathology and speculum exam.

KEYWORDS: spontaneous abortion - MRI - first trimester bleeding

\section{Introduction}

Vaginal bleeding in the first trimester ( 0 to 13 6/7 weeks) occurs in approximately 30 percent of all pregnancies [1,2]. The four major sources of non traumatic first trimester bleeding are ectopic pregnancy, implantation of the fertilized egg in the lining of the uterus, cervical or uterine pathology, and miscarriage. Spontaneous abortions, or miscarriages, are the most common cause of first trimester bleeding, presenting with bilateral lower abdominal pain and are diagnosed by ultrasound (US) findings [3]. Here, we report a 25 year old female with vaginal bleeding, bilateral abdominal pain, and normal transvaginal US findings on admission. Follow-up magnetic resonance imaging (MRI) in the emergency department (ED) captured the active passage of products of conception (POC), confirmed by pathology and speculum exam.

\section{Case Report}

A 25 year old G1 P0010 pregnant female at 9+4 weeks estimated gestational age via ultrasound presented to our ED with complaints of bilateral lower abdominal pain and vaginal spotting for the past two days. Patient reported severe, cramping, bilateral lower quadrant $10 / 10$ abdominal pain that was aggravated by movement. The bright red spotting has been constant for the past two days, but patient passed a 3-4 cm clot prompting the ED visit. Pelvic exam showed small blood clots in the posterior vaginal canal without any lesions and a normal closed cervix. Initial transvaginal ultrasound showed an unremarkable live intrauterine pregnancy without free fluid. A pelvic MRI was ordered for further evaluation as the patients pain continued after a normal pelvic exam and ultrasound findings. MRI exam demonstrated heterogeneous, T2 isointense/ hyperintense signal material extending from the uterine cavity and protruding into the vaginal canal, consistent with active passage of POC. The patient was removed from the scanner and subsequently passed the retained POC, confirmed by pathology and speculum exam.

\section{Discussion and Conclusion}

Spontaneous Abortion (SA) is defined as a clinically recognized pregnancy loss before the $20^{\text {th }}$ week of gestation $[1,2]$. SA is prevalent in 20 percent of all pregnancies and it is subdivided into threatened, inevitable, incomplete, missed, complete, and recurrent spontaneous abortion. Some of the best documented risk factors for SA are advanced maternal age, previous spontaneous abortion, and maternal smoking. Our patient is a young non-smoker with a previous uncomplicated abortion at 7 weeks increasing her risk for the current SA. The risk of abortion in future pregnancy is approximately 20 percent after one miscarriage and almost 30 percent after two miscarriages [3]. SA typically present with vaginal bleeding or crampy pelvic pain and the speculum exam is used to confirm bleeding from the uterus. The speculum exam also evaluates for a dilated cervix and active passage of POC in the cervix or vaginal canal. Another common feature in SA is a small for gestational age uterus that can be assessed with

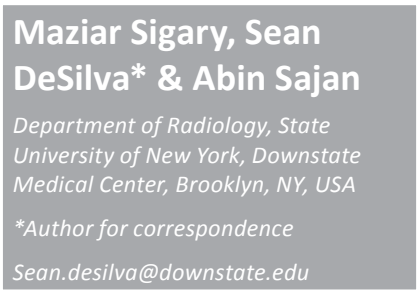


a bimanual pelvic exam. Although our patient presented with the classic severe bilateral pelvic pain, along with vaginal bleeding, the physical exams showed a closed cervix without any fetal tissue in the vaginal canal and an appropriately sized uterus.

Transvaginal ultrasound is the most important diagnostic test with a suspected spontaneous abortion [4]. It evaluates for fetal cardiac activity, size of the gestational sac, presence of a yolk sac, the fetal heart rate, and crownrump length [5]. Diagnostic findings of SA are crown-rump length (CRL) greater than or equal to $7 \mathrm{~mm}$ with no heartbeat, mean sac diameter greater than or equal to $25 \mathrm{~mm}$ with no visualized embryo, absence of embryo with heartbeat greater than or equal to two week after a scan that showed a gestational sac without a yolk sac, and absence of embryo with heartbeat greater than or equal to eleven days after a scan that showed a gestational sac with a yolk sac [5-7]. While these are definitive signs, findings suspicious for SA include: sudden loss of heartbeat or embryo after previously noted normal findings, empty amnion, enlarged yolk sac, and small gestational sac. The majority of SA in the first trimester are caused by fetal factors like chromosomal abnormalities and mosaicism [8]. Studies have also found that structural uterine anomalies like septate uterus and submucosal leiomyoma can also cause early pregnancy termination. Other maternal causes include systemic diseases like endocrinopathies and hypercoagulable states which are dangerous for the placenta [9]. Our patient had no history of any systemic diseases and her transvaginal US in the ED demonstrated a normal physiologic uterus with a fetal heart rate around 172, CRL of 24 , and a normally visualized gestational and yolk sac without a subchorionic hematoma, which has been shown to increase the risk for SA (FIGURE 1) [10].

Although MRIs are not involved in the SA workup, an MRI study was ordered in the ED in

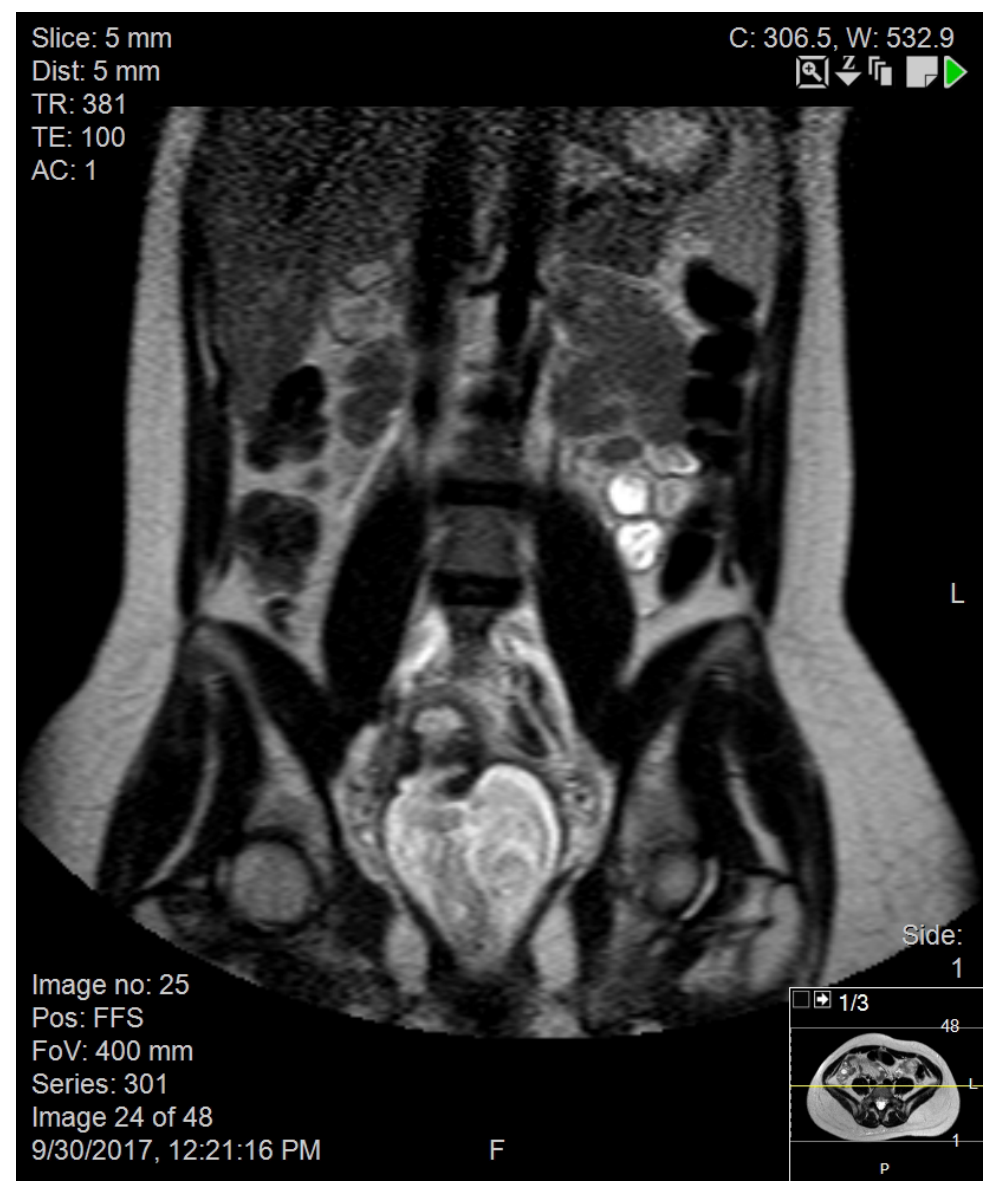

Figure 1. CRL of 24, and a normally visualized gestational and yolk sac without a subchorionic hematoma, which has been shown to increase the risk for SA. 

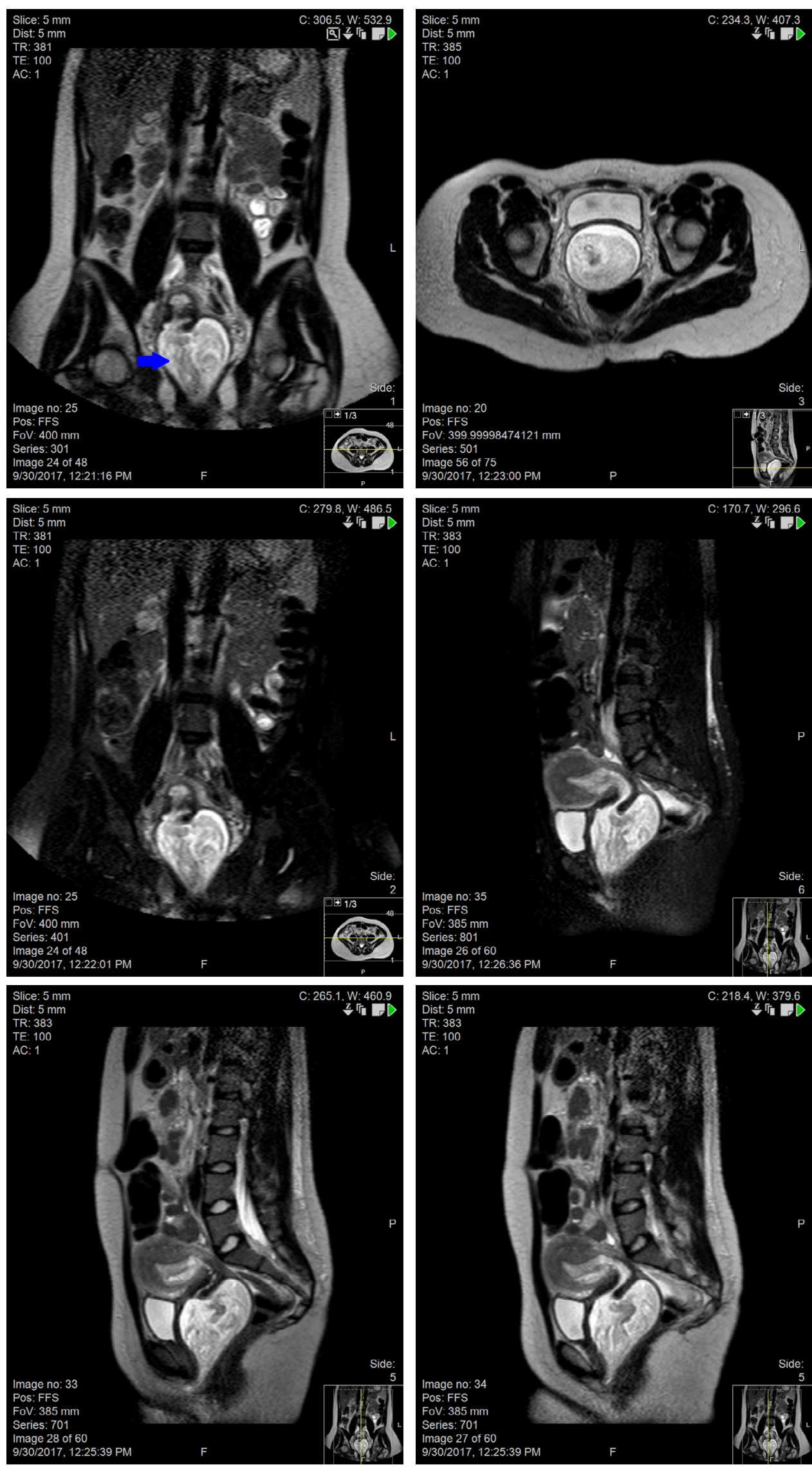


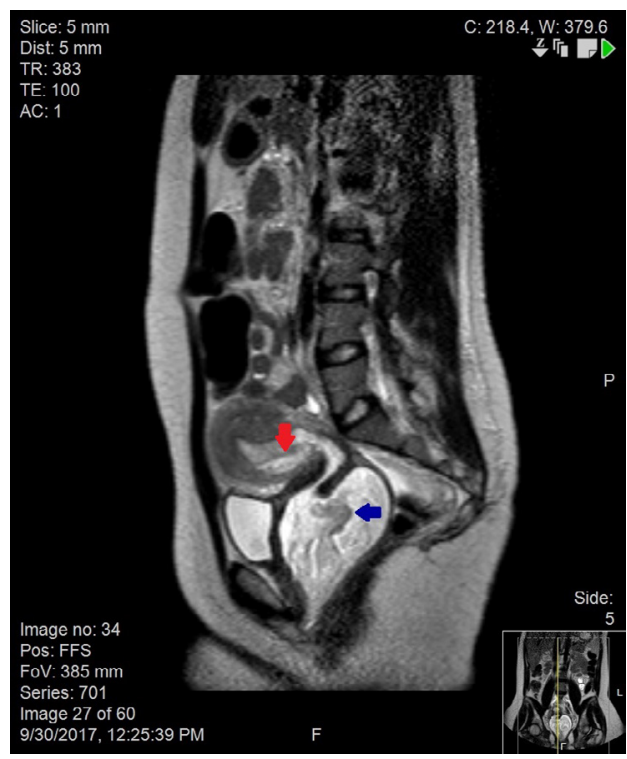

Figure 2. Sagittal MRI demonstrated no definite fetal parts and showed heterogeneous, T2 isointense/ hyperintense signal material extending from the uterine cavity (red arrow) and protruding into the vaginal canal (blue arrows), consistent with active passage of POC.

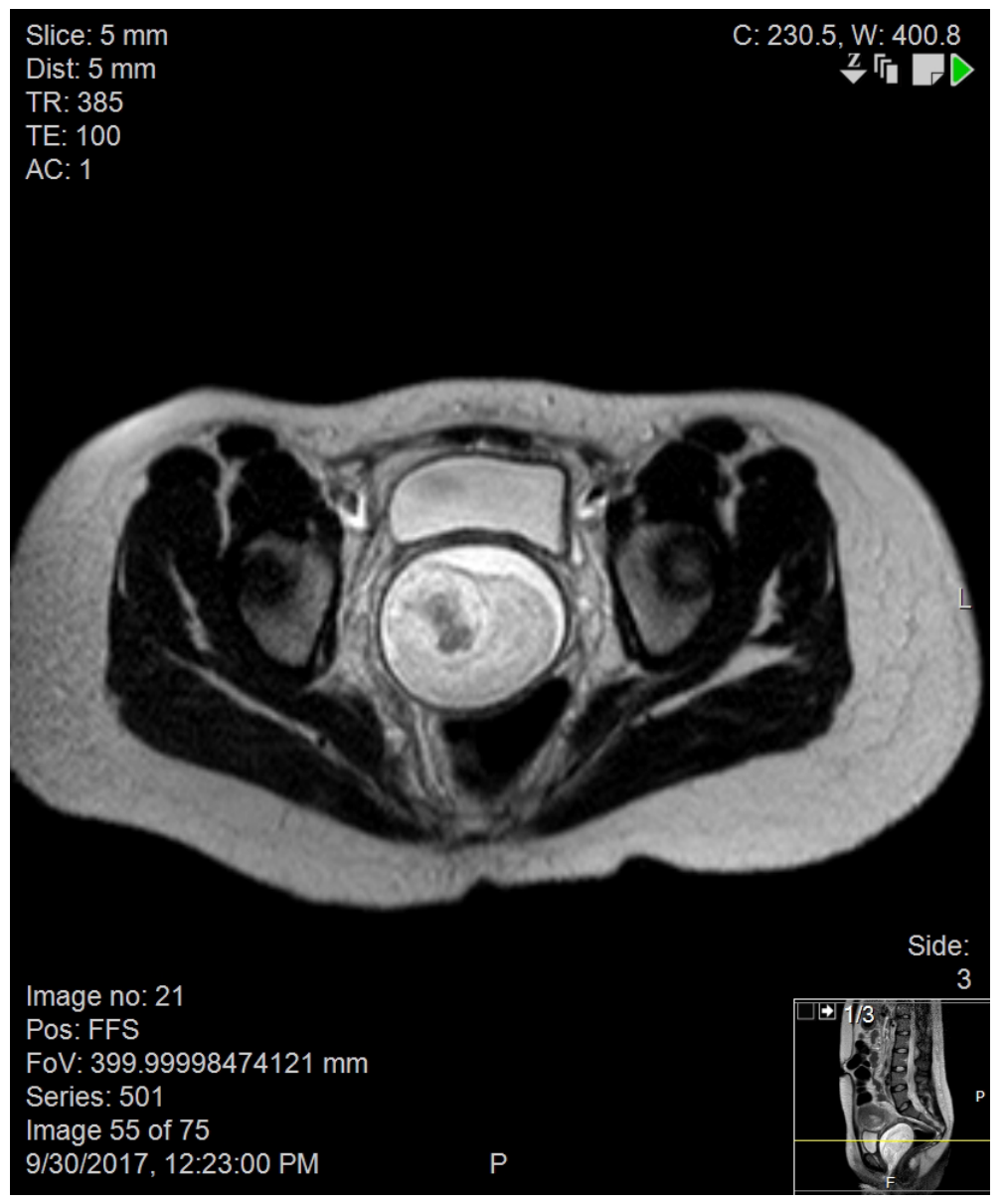

Figure 3. Follow-up transvaginal ultrasound demonstrated no intrauterine gestation or retained POC. 
the background of otherwise normal findings to asses for a potential appendicitis, which has been commonly reported during pregnancy in the literature [11]. Sagittal MRI demonstrated no definite fetal parts and showed heterogeneous, T2 isointense/hyperintense signal material extending from the uterine cavity (red arrow) and protruding into the vaginal canal (blue arrows), consistent with active passage of POC
(FIGURE 2). The patient was removed from the scanner and subsequently passed the retained POC, confirmed by pathology and speculum exam. Follow-up transvaginal ultrasound demonstrated no intrauterine gestation or retained POC (FIGURE 3). The imaging findings in this case of spontaneous abortion are remarkable in that it catches the moment of active passage of POC.

\section{REFERENCES}

1. Nanda K, Peloggia A, Nanda G et al. Expectant care versus surgical treatment for miscarriage. Cochrane. Database. Syst. Rev. 3, 1-49 (2012).

2. Regan L, Rai R. Epidemiology and the medical causes of miscarriage. Best. Pract. Res. Clin. Obstet. Gynaecol. 14, 839-854 (2000).

3. Regan L, Braude PR, Trembath PL. Influence of past reproductive performance on risk of spontaneous abortion. BMJ. 299, 541-545 (1989)

4. Dighe M, Cuevas C, Moshiri M et al.
Sonography in first trimester bleeding. J. Clin. Ultrasound. 36, 352-366 (2008).

5. Tongsong T, Wanapirak C, Srisomboon J et al. Transvaginal ultrasound in threatened abortions with empty gestational sacs. Int. J. Gynecol. Obstet. 46, 297-301 (1994).

6. Deaton, JL, Honore GM, Huffman CS et al. Early transvaginal ultrasound following an accurately dated pregnancy: The importance of finding a yolk sac or fetal heart motion. Human. Reprod. 12, 2820-2823 (1997).

7. Bourne T, Bottomley C. When is a pregnancy nonviable and what criteria should be used to define miscarriage?. Fertil. Steril. 98, 1091-1096 (2012)

8. Levy B, Sigurjonsson S, Pettersen B et al. Genomic Imbalance in Products of Conception. Obstet. Gynecol. 124, 202-209 (2014).

9. Stankiewicz P. NAD deficiency, congenital malformations, and niacin supplementation. $N$. Engl. J. Med. 377, 544-552 (2017).

10. Pearlstone M, Baxi L. Subchorionic Hematoma. Obstet. Gynecol. Surv. 48, 55-68 (1993).

11. Thompson M, Kudla A, Chisholm C. Appendicitis during Pregnancy with a normal MRI. West. J. Emerg. Med. 15, 652-654 (2014). 